\title{
VEREINSRECHT
}

\section{Mehrheit ist nicht immer Mehrheit}

- Dietmar Weidlich

\begin{abstract}
"Die Mitgliederversammlung fasst ihre Beschlüsse mit relativer Mehrheit. "Was sich in Vereinssatzungen einfach liest, kann im Konfliktfall kompliziert werden. Wann ist eine Mehrheit eine Mehrheit?
\end{abstract}

Immer wieder kommt es in Mitgliederversammlungen bei Abstimmungen mit knappen Mehrheiten aufgrund unklarer Satzungsformulierungen zu Unsicherheiten, insbesondere wenn wichtige Entscheidungen anstehen. Was bedeuten die Begriffe »einfache«, »relative «, »qualifizierte « oder »absolute « Mehrheit? Welche »Mehrheit« ist für eine Zweckänderung erforderlich? Werden Enthaltungen zur Feststellung der Mehrheit mitgezählt? Auch die Frage der Beschlussfähigkeit ist häufig unklar. Was gilt, wenn bei Versammlungsbeginn genügend Mitglieder anwesend sind, aber vor der Abstimmung den Saal verlassen? Sind die mit der erforderlichen Mehrheit gefassten Beschlüsse (z. B. Neuwahl eines Vorstands) dann trotzdem wirksam?

\section{Beschlussfähigkeit}

Die in der Praxis übliche Feststellung der Beschlussfähigkeit $» z u$ Beginn der Versammlung « ist irreführend, weil sich erfahrungsgemäß gerade bei längeren Versammlungen die Zahl der Teilnehmer ständig ändern kann. Daher ist - zumindest bei einer grenzwertigen Anzahl der Teilnehmer im Zweifel - die Beschlussfähigkeit vor der Abstimmung über jeden Beschlussgegenstand festzustellen, auch wenn dies mühsam ist. Die Feststellung der Beschlussfähigkeit bedeutet zweierlei: Reicht die Zahl der erschienenen stimmberechtigten Mitglieder aus, um über den Beratungsgegenstand abzustimmen (die nicht stimmberechtigten
Mitglieder werden also trotz Teilnahmerechts nicht mitgezählt) und zum anderen: Darf über den Beratungsgegenstand überhaupt abgestimmt werden, weil er beispielsweise keinem Tagungsordnungspunkt zugeordnet werden kann.

Das Bürgerliche Gesetzbuch (BGB) schreibt keine Mindestteilnehmerzahl für die Beschlussfähigkeit vor, sondern stellt nur auf die erschienenen Mitglieder ab (\$§ 32 Abs. 1 S. 1 BGB). Sofern die Satzung nicht - wie im Regelfall - einen bestimmten Prozentsatz oder eine feste Anzahl von erschienenen Mitgliedern verlangt, liegt Beschlussfähigkeit vor, wenn die Versammlung ordnungsgemäß einberufen wurde und zumindest ein stimmberechtigtes Mitglied anwesend ist.

Verlassen stimmberechtigte Teilnehmer den Saal und ist dadurch keine Beschlussfähigkeit mehr gegeben, muss der Sitzungsleiter die Versammlung sofort förmlich schließen, sofern nicht mit einem alsbaldigen Erscheinen der zur Beschlussfähigkeit erforderlichen Mitglieder zu rechnen ist. Werden trotzdem Beschlüsse gefasst (weil z. B. der Versammlungsleiter nicht bemerkt hat, dass die anfängliche Beschlussfähigkeit mittlerweile nicht mehr besteht), sind diese nichtig, Wahlen sind zu wiederholen.

\section{Die »erforderliche Mehrheit»}

Liegt Beschlussfähigkeit vor, erfolgt nach der Abstimmung die Auszählung der Stimmen, wobei (nach herrschender Meinung) nur Ja- und Nein- Stimmen berücksichtigt werden. Stimmenthaltungen und ungültige Stimmen werden zur Feststellung der Mehrheit nicht mitgezählt (BGH 83, 35).

Eine »einfache Mehrheit « ist also gegeben, wenn die Zahl der Ja-Stimmen

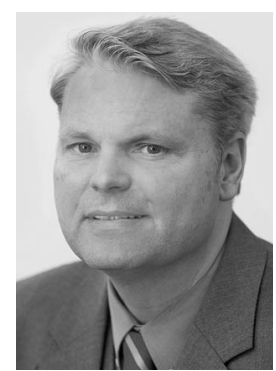

Rechtsanwalt

Dietmar Weid-

lich berät seit eineinhalb Jahrzehnten als Syndikusanwalt Einrichtungen der Sozialwirtschaft bei Neugründungen und Umstrukturierungen sowie Kooperationen und Fusionen. Er hat sich neben dem Gesellschaftsrecht vor allem auf das Vereinsund Stiftungsrecht spezialisiert. E-Mail dietmar.weidlich@ curacon.de

größer ist als die Zahl der Nein-Stimmen, unabhängig davon, wie viele Mitglieder sich enthalten. Die Anzahl der anwesenden stimmberechtigten Mitglieder ist also nur entscheidend für die Frage der Beschlussfähigkeit, nicht aber für die Mehrheit. Bei Stimmengleichheit ist der Beschluss nicht zustande gekommen.

Hingegen gilt ein Beschluss als gefasst, wenn bei der Stimmenauszählung zwar Fehler gemacht wurden, dennoch aber zweifelsfrei feststeht, dass sich auch ohne Berücksichtigung der fehlerhaft gezählten Stimmen die erforderliche Mehrheit ergibt (BayObLG MDR 1990, 449).

Natürlich kann die Satzung abweichende Regelungen vorsehen, indem sie beispielsweise Stimmenthaltungen und ungültige Stimmen als Nein-Stimmen wertet oder bei besonders wichtigen Entscheidungen die Anwesenheit eines bestimmten Quorums verlangt.

\section{Stimmrechte von Mitgliedskörper- schaften}

Grundsätzlich hat jedes Mitglied gleich, ob natürliche oder juristische Person - nur eine Stimme (Grundsatz der »Gleichheit aller Vereinsmitglieder «), jedoch kann die Satzung bestimmten Mitgliedern (z. B. Körperschaften) ein erhöhtes (mehrfaches) Stimmrecht für alle oder bestimmte Angelegenheiten zuweisen. Unabhängig wie viele Vertreter eine solche Körperschaft in die Versammlung entsenden darf, kann sie jedoch die ihr 
zustehenden Stimmen nur einheitlich abgeben (»Verbot der Stimmrechtsspaltung «). Gegen dieses Verbot wird - mangels Kenntnis - häufig bei Vereinen verstoßen, die eine Vielzahl von Körperschaften mit unterschiedlich vielen Stimmen haben und durch mehrere Personen in der Mitgliederversammlung vertreten werden.
Das höchste denkbare Quorum, nämlich die »Einstimmigkeit «, verlangt das Gesetz bei Änderungen des Vereinszwecks (§ 33 Abs. 1 S. 2 BGB). Hierbei müssen alle erschienenen Mitglieder mit Ja stimmen, jede Enthaltung oder ungültige Stimme verhindert die Einstimmigkeit. Nicht erschienene Mitglieder können später

\section{"Nach dem Gesetz kann Beschluss- fähigkeit auch bei nur einem erschienenen Vereinsmitglied vorliegen «}

\section{Die verschiedenen Arten von Mehrheiten}

Neben der einfachen Mehrheit findet sich in Satzungen (nicht aber im Gesetz) gelegentlich die »relative (oder verhältnismäßige) Mehrheit «, die vorliegt, wenn einer von mehreren Beschlussanträgen die meisten JaStimmen erhält. Dies kommt häufig bei Personalentscheidungen vor, wobei derjenige gewählt ist, der die meisten Stimmen auf sich vereinigt. Fehlt eine solche Regelung in der Satzung und erreicht keiner der Kandidaten zumindest die "einfache Mehrheit« (§ 32 Abs. 1 S. 3 BGB), ist die Wahl zu wiederholen.

Eine »absolute Mehrheit « bedeutet im Gegensatz zur relativen Mehrheit nichts anderes als die einfache Mehrheit, wobei die Satzung klarstellen muss, ob damit - wie im gesetzlichen Regelfall - die Mehrheit der erschienenen oder die Mehrheit aller stimmberechtigten Mitglieder gemeint ist. Fehlt eine solche Klarstellung, gilt die gesetzliche Regelung.

Häufiger findet sich hingegen eine »qualifizierte Mehrheit « bei wichtigen Entscheidungen. Hierbei handelt es sich um eine größere Mehrheit als die »einfache«, erreicht aber nicht die Einstimmigkeit. So sieht das Gesetz beispielsweise für Satzungsänderungen eine Dreiviertel-Mehrheit der erschienenen Mitglieder vor $(\S 33$ Abs. 1 S. 1 BGB), jedoch kann die Satzung dafür auch jede andere qualifizierte Mehrheit vorsehen (z. B. die Hälfte oder zwei Drittel aller Vereinsmitglieder). In diesem Fall werden auch Stimmenthaltungen und ungültige Stimmen zur Feststellung der Mehrheit mitgezählt, sofern die Satzung nichts anderes regelt. schriftlich zustimmen. Allerdings kann auch hierbei die Satzung andere qualifizierte Mehrheiten vorsehen. Eine »Zweckänderung « liegt allerdings nur vor, wenn andere als die bisher in der Satzung verankerten Hauptziele verfolgt oder bislang untergeordnete Ziele künftig zum Hauptzweck gemacht werden sollen, also der »oberste Leitsatz des Vereins « in seinem Wesen verändert wird. Dies ist nicht der Fall bei einer bloßen sprachlichen Anpassung der Vereinszwecke an die veränderten Verhältnisse.

Soll die bisherige Regelung zur Änderung des Vereinszwecks im Rahmen einer anstehenden Satzungsänderung im Hinblick auf die erforderliche Mehrheit verändert werden, bedarf dies bei der Abstimmung der bislang vorgeschriebenen Mehrheit. War dies also bislang in der Satzung nicht ausdrücklich geregelt, ist gemäß der gesetzlichen Vorgabe $(\S 33$ Abs.1 S. 2 BGB) für eine Herabsetzung ebenfalls Einstimmigkeit erforderlich.

\section{Fazit}

Die Satzung sollte sowohl im Hinblick auf die Beschlussfähigkeit als auch auf die erforderlichen Mehrheiten präzise Regelungen enthalten. So kann beispielsweise für einfache Satzungsänderungen ein niedrigeres Quorum gewählt werden als für Zweckänderungen oder die Auflösung des Vereins. Auch sollte klargestellt werden, ob zur Feststellung der Mehrheit Enthaltungen und ungültige Stimmen mitzählen oder nicht und ob die Mehrheit der erschienenen oder die Mehrheit aller Vereinsmitglieder gemeint ist. Beide Regelungsgegenstände können für wichtige Entscheidungen kombiniert werden.

\section{Soziale Dienste in Europa}

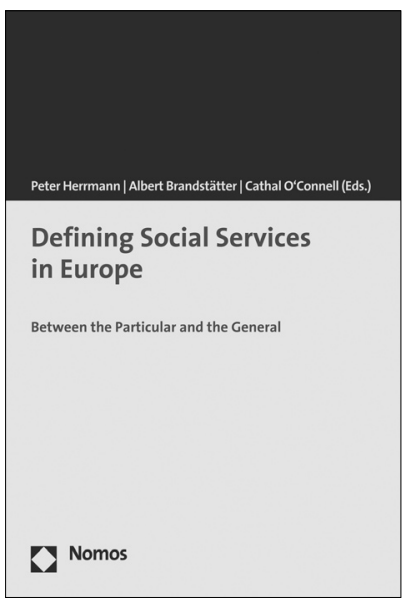

\section{Defining Social Services in Europe}

Between the Particular and the General

Herausgegeben von Dr. Peter Herrmann, Albert Brandstätter und Dr. Cathal O'Connell 2007, 276 S., brosch., 44,- $€$, ISBN 978-3-8329-2883-4

Soziale Dienstleistungen des Allgemeinen Interesses stehen hoch im Kurs der EU-politischen Debatte um die Wettbewerbsfreiheit. Aber können Dienste für jene, die aus dem Wettbewerb fallen, überhaupt dem Wettbewerbsrecht folgen? Und leisten sie nicht einen Dienst gerade auch für die Gesellschaft, anstatt nur für hilfebedürftige Individuen da zu sein?

Der Band untersucht verschiedene Aspekte der Qualität sozialer Dienste aus theoretischer und praktischer Sicht.

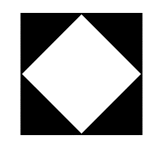

Nomos

Bitte bestellen Sie im Buchhandel oder versandkostenfrei unter $\downarrow$ www.nomos-shop.de 